\title{
Sensitivity Enhancement of Ag-Ni Functional Bimetallic SPR Sensor Using Black Phosphorus in the Visible Region
}

prem kumar ( $\sim$ premhawal@gmail.com )

Glocal University https://orcid.org/0000-0003-3339-8724

Rajeev Kumar

Glocal University

O. K. Harsh

Glocal University

Vikram Singh

Glocal University

\section{Research Article}

Keywords: SPR Sensor, Sensitivity, Detection accuracy, Figure of merit, FWHM

Posted Date: May 24th, 2021

DOI: https://doi.org/10.21203/rs.3.rs-518410/v1

License: (c) (i) This work is licensed under a Creative Commons Attribution 4.0 International License.

Read Full License 


\title{
Sensitivity enhancement of Ag-Ni functional bimetallic SPR sensor using black phosphorus in the visible region
}

\author{
Prem Kumar ${ }^{1}$, Rajeev Kumar ${ }^{2}$, O. K. Harsh ${ }^{3}$, Vikram $\mathrm{Singh}^{4}$ \\ ${ }^{1,2,3,4}$ Electronics and Communication Engineering, Glocal University, Saharanpur, UP, India. \\ Email Id: prem@theclocaluniversity.in, premhawal@gmail.com (P. Kumar), \\ rajeevprakr@gmail.com (R. Kumar), oharsh@gmail.com ( O. K. Harsh), tomar.vs@gmail.com \\ (V. Kumar)
}

\begin{abstract}
In this seminal, a highly sensitive bimetallic layer for surface plasmon resonance (SPR) sensor applications is proposed with the BK7 prism, silver-nickel (Ag-Ni) bimetallic films, and hybrid structure of black phosphorus layer under angular interrogation technique. Sensitivity, minimum reflectivity $\left(\mathrm{R}_{\mathrm{min}}\right)$, DA, and FoM are the criterion for the sensing applications. By tuning these parameters, the maximum sensitivity of $347^{\circ} / \mathrm{RIU}$ is obtained with two-dimensional (2D) black phosphorus and the optimized thickness of the Silver layer at $633 \mathrm{~nm}$ wavelength. 2D black phosphorus (BP) has attracted immense interest from the research community because of its unique properties like high charge density, direct bandgap, and better binding to the various molecules. The noble bimetallic layer-based SPR sensors have improved the sensor performance due to their exciting properties of large optical field enhancements follow on to the strong scattering and absorption of light. Herein, the Black Phosphorus-based SPR sensor is optimized with the thickness variation of the Silver layer due to ease of synthesis, a high degree of surface functionalization, and tunable Physico-chemical properties to the various sensing-medium refractive indices ranging from 1.330 to 1.335 . Although there are many analogous sensors reported, limited attention has been paid to propose one, which exhibits the higher sensitivity, better DA, and superior FoM. The increased sensitivity of bimetallic layer and 2D, Black Phosphorus-based sensor providing promising applications in biosensing.
\end{abstract}

Keyword: SPR Sensor, Sensitivity, Detection accuracy, Figure of merit, FWHM.

\section{Research Highlights}

- Proposed SPR Sensor: BK7 Prism- Ag-Ni-Black Phosphorous-Sensing medium.

- Black Phosphorous layer plays important role in enhancing the sensitivity.

- The Highest Sensitivity ( $\left.347^{\circ} / \mathrm{RIU}\right)$ is achieved for the proposed SPR Sensor.

- If used $20 \mathrm{~nm}$ thickness Nickel layer in the proposed SPR sensor, the Sensitivity is reached up to $476^{\circ} / \mathrm{RIU}$.

- Detailed numerical analysis performed in angular interrogation mode.

\section{Novelty of the Proposed Work}


- This work focuses on designing SPR sensor for the detection of biomolecules.

- Firstly, we numerically investigated the Black Phosphorous layer for the proposed SPR sensor.

- The proposed SPR sensor demonstrates the Sensitivity due to the Black Phosphorous layer, which acts as a polarizer by manipulating the propagation of p-polarized light.

- Black Phosphorous 2D material used as the metal, it has sensing ability for detection of biomolecules, and we have utilized it as a bio-recognition element layer.

- After citing exemplary instances from the literature on SPR sensor, we found that till date none of the researchers have designed used as bimetallic layer SPR sensor based on Black Phosphorous layers to enhance figure of merit.

- Although we have not verified the distinct performance of SPR sensor experimentally, this work provides guidelines for designing a highly SPR device suitable for biosensing (sensing) applications.

- The maximum Sensitivity (347\%/RIU) is achieved for the proposed SPR Sensor.

- If used $20 \mathrm{~nm}$ thickness Nickel layer in the proposed SPR sensor, the Sensitivity is reached up $476^{\circ} / \mathrm{RIU}$.

\section{Introduction}

Surface Plasmon Resonance (SPR) technology has attracted the attention of several scientific communities due to its key advantage over traditional biosensors, including increased sensitivity, ease to use, lower cast, and label-free and real-time detection (Biswajit et al. 2021). Temperature and pressure measurements (Mollah et al. 2020), plasmonic detector (Mollah et al. 2020), optical bi-stability (Moznuzzaman et al. 2020), formalin (Sharma et al.2010), human-blood group (Lam et al. 2005), glucose (Lam et al. 2005), food and environmental (Shankaran et al. 2007), gassensing (Mishra et al 2014), bacteria (Maurya et al. 2018), urine (Menon et al. 2018), DNAhybridization (Rahman et al. 2017), and living cell analysis (Yanase et al.2019), etc. are just a few of the various application for the SPR based sensor. The SPR observes the biomolecular interaction within a limited axial region on the sensor surface. When incident light on the sensor surface excites the surface plasmon, a sensing field is generated. The total reflection configuration, at the resonance dip condition, is formed in the angular reflection spectrum due to energy dissipation into the metallic layer or scattering on the sensor surface. It is appropriate for detecting the molecules with its higher shift angle and straightforward fabrication technique; it is also dependent on the surface plasmon and generates the evanescent wave by the ' $p$ ' polarized light. The SPR biosensor depends on surface plasmon resonance waves, induced by evanescent waves, which are the result of attenuated total reflection (ATR), generated at a prism-metal interface. When the evanescent wave is matched to the wave-vector of surface plasmon, a sharp reflectivity curve is observed. In the current scenario, SPR biosensors have become a research hotspot because of sensor performance for imperative behalf of biological sensing and exploring 
the performance through improvement mechanisms. The various structures of the SPR biosensors have been vigorously studied in recent years in order to obtained maximum sensitivity and lower limit detection (Yuan et al. 2018, Wu et al. 2016, Kumar et al 2018). Shalabney et al. (2011) theoretically discussed a variety of methods to enhance the sensor performance, including optimizing SPR sensitive metal layers, considering the impact of prismindex, dispersion, adding nano-dielectric layers, and incorporation of the gratings on top of the metals. Dyankov et al. (2012), have proposed using a bimetallic film to improve the sensitivity of the SPR sensor. Kashyap et al. (2019) and Chen et al. (2016) discuss and comparison to a singlemetal-based sensor proposed a bimetallic (Ag-Au) based sensor with improved sensitivity and reduced FWHM with higher detection accuracy. However, for practical applications, the performance of SPR sensors requires to be improved. In order to establish the reliability and usefulness of an SPR based sensor, the reflectivity analysis plays a vital role, which dependent on several parameters namely wavelength, incident light, metal, dielectric constant, refractive indices, and sensing medium. The potential metals for SPR sensor applications are known as gold $(\mathrm{Au})$, Silver $(\mathrm{Ag})$, aluminum $(\mathrm{Al})$, copper $(\mathrm{Cu})$, indium $(\mathrm{In})$, and sodium $(\mathrm{Na})$ for sensing. Gold and silver are mostly used in SPR sensors because of great stability, durability, biocompatibility, higher sensitivity, and a great figure of merit (FoM) as compared to other contenders (Mitsushio et al. 2006; Rifat et al. 2016). The Ag-based SPR sensor provides narrow spectral width because it is chemically inactive and highly vulnerable to oxidation. However, Au-based sensors are also recognized for high sensitivity due to their chemical inertness. Recently, nickel (Ni) has been explored for bimetallic configuration because it has excellent magneto-optical merit and exhibits an enhanced sensing signal. Additionally, Ni is chemically low active, lower in cost, and lower in cost. Hence, the bulk uses of Ni can reduce the cost of SPR sensors in comparison to other metals for commercial-scale production.

In conjunction with this now, the researcher community is working extensively to explore the other possible biosensors with improved sensitivity. Recently, various 2D materials have been investigated including transition metal dichalcogenides (TMDs) (Wang et al. 2012), graphene (Abbas et al. 2015), black phosphorus (Yasaei et al.2015), which is used on the top of plasmonic supporting materials of SPR sensor applications. Several 2D materials have been considered as potential chemical-sensing materials, owing to their exceptional physicochemical and merits onto targeted molecules, due to tunable bandgap, adsorption energy, and compatible physical properties. For example, graphene has strong mechanical stability and higher electrical conductivity. It possesses a low signal-to-noise ratio, and a fine response to the target analytes. Recently, black-phosphorus (BP) has been introduced in the 2D materials family as a favorable new material for sensing applications. BP exhibits better molecular adsorption energy than other 2D materials such as graphene and TMDs and extraordinary electrical properties like high charge density, direct bandgap, optical and better binding of molecules. Lately, there are many reports are on BP-based SPR sensors with considerable sensitivity. Sharma et al. (2007), discussed the various bimetallic combinations of SPR sensors based on $\mathrm{Ag}, \mathrm{Au}, \mathrm{Cu}$, and $\mathrm{Al}$ and successfully demonstrated that the best combination of bimetallic $\mathrm{Ag}-\mathrm{Au}$ and $\mathrm{Cu}-\mathrm{Au}$ provides the maximum 
sensitivity than other combinations. Srivastava et al. (2020), theoretically proposed an SPR sensor-based Au-MXene-WS 2 -Black Phosphorus with enhanced sensitivity of $\sim 190.22^{\circ} / \mathrm{RIU}$ at a wavelength $(K) 633 \mathrm{~nm}$. Vibisha et al. (2020), also theoretically discussed the use of a few TMDs material over a bimetallic layer to obtain the higher sensitivity and FWHM particularly with optimized Copper layer and Nickel layer. Wu et al. (2017), design a hetero-structure (fewlayer BP and Graphene/TMDs) based SPR sensor, and demonstrated the sensitivity of 279 \%RIU. Maharana et al. (2012), proposed a bimetallic (Al-Au) based SPR sensor along with chalcogenide prism and obtained FWHM, which is exhibited 6-times narrower than a singlemetal layer SPR sensor. As well, the electric field increase of the Al-Au configuration is fifty times greater at the metal analytes interface.

In the present work, the bimetallic layer-based SPR sensor has been designed and investigated using 2D black phosphorus (BP) for sensing applications. The sensor sensing performance is established by characterizing the reflectance curve of the SPR biosensor having Silver (Ag), Nickel (Ni), and 2D BP systematically. Hence, the sensitivity, minimum reflectance, DA, and FoM are evaluated. Further, the numerical modeling to simulate results has been carried out analytically for the proposed SPR sensor for commercial-scale prototyping.

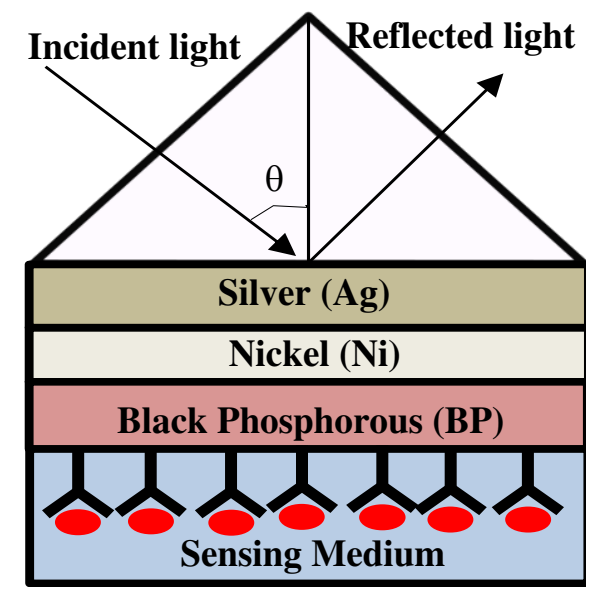

Fig.1 Proposed SPR Sensor

\section{Numerical modeling of proposed SPR sensor}

\subsection{Proposed SPR-Sensor description}

We have systematically investigated the various proposed bimetallic SPR biosensor consisting of 2D BP and sensing layers as depicted in Fig.1. The proposed design SPR structure works in the visible range at a wavelength of $633 \mathrm{~nm}$. Herein, the $2 \mathrm{D}$ material $(\mathrm{BP})$ is employed to enhance the adsorption capacity of the sensor, which is more beneficial to obtain maximum sensitivity and enhance biocompatibility. All the performance parameters are analyzed and simulated using the transfer-matrix-method (TMM) and Fresnel equation. The investigated SPR sensor analysis 
indicates that the Ag-Ni-BP-SM optimum system configuration, then the existing bimetallic combination and result in better sensing performance.

\subsection{Proposed Structure Layer Configuration}

The proposed SPR sensor is analyzed for the set-up as shown in Fig.1, wherein the light incident on a BK7 prism. The BK7 prism, which has a refractive index (RI) of 1.5151, is the first layer (Vibisha et al. 2020). The $2^{\text {nd }}$ and $3^{\text {rd }}$ layers are $\mathrm{Ag}, \mathrm{Au}$, and $\mathrm{Ni}$ metal film. The dielectric constant of both metals ( $\mathrm{Ag}$ and $\mathrm{Ni}$ ) has been mentioned below. The RI is calculated by the Drude-model formula (Maharana et al. 2012):

$\mathrm{n}_{\text {silver }}=\left(\varepsilon_{\mathrm{r}}+\mathrm{i} \varepsilon_{\mathrm{i}}\right)^{\frac{1}{2}}=\left(1-\frac{\lambda^{2} \lambda_{\mathrm{c}}}{\lambda_{\mathrm{p}}^{2}\left(\lambda_{\mathrm{c}}+\mathrm{i} \lambda\right)}\right)^{\frac{1}{2}}$

Where $\lambda_{c}$ and $\lambda_{p}$ represents the collision and plasma wavelength for Silver, Gold, and Nickel as shown in table 1 at $\lambda=0.633 \mu \mathrm{m}$.

Table 1: Collisions and plasma wavelength of Silver, Gold, and Nickel calculated by the Drudemodel at $\lambda=633 \mathrm{~nm}$.

\begin{tabular}{|l|l|c|l|}
\hline S.No. & Materials & Collisions wavelength $(\boldsymbol{\lambda} \mathbf{c})$ & Plasma wavelength $(\boldsymbol{\lambda} \mathbf{p})$ \\
\hline 1 & Silver $(\mathrm{Ag})$ & $1.7614 \times 10^{-5}$ & $1.4541 \times 10^{-7}$ \\
\hline 2 & Gold $\mathrm{Au})$ & $8.9342 \times 10^{-6}$ & $1.6826 \times 10^{-7}$ \\
\hline 3 & Nickel $\mathrm{Ni})$ & $2.8409 \times 10^{-5}$ & $2.5381 \times 10^{-7}$ \\
\hline
\end{tabular}

The third layer is black phosphorus (BP) of thickness as $0.53 \mathrm{~nm}$ and its RI is $5.3+0.01 \mathrm{i}$ (Srivastava et al. 2020).

\subsection{Proposed SPR sensor modeling}

In order to calculate the reflection-coefficient expression for the ' $p$ ' polarized light. The TMM is discussed below for the N-layer model. This method is very accurate because it has not contained an approximation. The different medium layer is characterized by the thickness $\left(\mathrm{d}_{\mathrm{k}}\right)$, permeability $\left(\mu_{\mathrm{k}}\right)$, dielectric constant $\left(\varepsilon_{\mathrm{k}}=\mathrm{n}^{2}\right)$, and RI $\left(\mathrm{n}_{\mathrm{k}}\right)$ for the kth layer. The tangential-field component at the $1^{\text {st }}$ boundary (at $\mathrm{Z}=\mathrm{Z}_{1}=0$ ) and $\mathrm{N}^{\text {th }}$ boundary (at $\mathrm{Z}=\mathrm{Z}_{\mathrm{N}-1}$ ) are related by the following relation (Srivastava et al. 2020).

$\left[\frac{\mathrm{X}_{1}}{\mathrm{Y}_{1}}\right]=M\left[\frac{X_{N-1}}{Y_{N-1}}\right]$

Where $X_{1}$ and $Y_{1}$ are the tangential electric-field component at the $1^{\text {st }}$ boundary layer. $X_{\mathrm{N}-1}$ and $\mathrm{Y}_{\mathrm{N}-1}$ are the tangential electric-field component at the $\mathrm{N}^{\text {th }}$ boundary layer. $\mathrm{M}$ is the combined layer system configuration's characteristic matrix. For the p-polarized light, $M$ is given as (Srivastava et al. 2020).

$M=\left[\begin{array}{ll}\mathrm{M}_{11} & \mathrm{M}_{12} \\ \mathrm{M}_{21} & \mathrm{M}_{22}\end{array}\right]=\prod_{\mathrm{k}=2}^{\mathrm{N}-1} \mathrm{M}_{\mathrm{k}}$

Where $\mathrm{M}_{\mathrm{k}}$ is the several layers. 
$M_{k}=\left[\begin{array}{cc}\cos \beta_{\mathrm{k}} & -\mathrm{i}\left(\sin \beta_{\mathrm{k}}\right) / \mathrm{q}_{\mathrm{k}} \\ -\mathrm{iq} \mathrm{q}_{\mathrm{k}} \sin \beta_{\mathrm{k}} & \cos \beta_{\mathrm{k}}\end{array}\right]$

Where $\mathrm{k}$ is a number ranging from 1 to $\mathrm{N}$.

Where, $\mathrm{q}_{\mathrm{k}}=\left(\frac{\mu_{\mathrm{k}}}{\varepsilon_{\mathrm{k}}}\right)^{\frac{1}{2}} \cos \theta_{\mathrm{k}}=\frac{\left(\varepsilon_{\mathrm{k}}-\mathrm{n}_{1}^{2} \sin ^{2} \theta_{1}\right)^{\frac{1}{2}}}{\varepsilon_{\mathrm{k}}}$

Here, $\beta_{\mathrm{k}}=\frac{2 \pi}{\lambda} \mathrm{n}_{\mathrm{k}} \cos \theta_{\mathrm{k}}\left(\mathrm{z}_{\mathrm{k}}-\mathrm{z}_{\mathrm{k}-1}\right)=\frac{2 \pi \mathrm{d}_{\mathrm{k}}}{\lambda}\left(\varepsilon_{\mathrm{k}}-\mathrm{n}_{1}^{2} \sin ^{2} \theta_{1}\right)^{\frac{1}{2}}$

The wavelength and incident angle of the incident-light is represented by $\lambda$ and $\theta_{1}$. The incidentangle at the BK7 prism, as depicted in Fig.1, is the parameter $\theta_{1}$. The reflection-coefficient is calculated by the two matrices. Both matrices are multiplied to calculate the transfer matrix (M) of the multilayer structure. Where $\mathrm{M}_{11}, \mathrm{M}_{12}, \mathrm{M}_{21}$, and $\mathrm{M}_{22}$, are calculated from equation (3). As a result, the p-polarized incident light's reflection-coefficient $(\mathrm{Rp})$ is as follows:

$R_{p}=\left|\frac{\left(M_{11}+M_{12} q_{N}\right) q_{1}-\left(M_{21}+M_{22} q_{N}\right)}{\left(M_{11}+M_{12} q_{N}\right) q_{1}+\left(M_{21}+M_{22} q_{N}\right)}\right|^{2}$

Where $\mathrm{q}_{1}$ and $\mathrm{q}_{\mathrm{N}}$ are corresponding terms for the $1^{\text {st }}$ layer (BK7-prism) and $\mathrm{N}^{\text {th }}$ layer(SM). Both terms are calculated from Eqn. ( 4 \&5) by putting in their respectively RI, dielectric constant, and incident angle values. The corresponding reflectance is given as:

$\mathrm{R}=\left|\mathrm{R}_{\mathrm{p}}\right|^{2}$

Where $\mathrm{Rp}$ is the reflection-coefficient of incident light and $\mathrm{R}$ is the reflectance intensity.

\subsection{Sensor performance parameter}

The following performance parameters defined for the proposed SPR sensor are sensitivity, FWHM, DA, and FoM. The MATLAB software is used to obtain all the performance parameters using mathematical modeling. Their definition is as follows:

Sensitivity ( $\mathrm{S})$ is defined as the difference between two resonance angles $\left(\Delta \theta_{\text {res }}=\theta_{2}-\theta_{1}\right)$ and sensing layer RI of 1.335-1.330 $\left(\Delta n_{s}=0.005\right)$ noted from the reflectance curve shows the SPR sensor is sensing capability (Srivastava et al. 2020).

$S=\frac{\Delta \theta_{\text {Res. }}}{\Delta n_{S}}($ Unit $=\mathrm{o} /$ RIU $)(\mathrm{Wu}$ et al. 2016)

Full Width at Half Maximum (FWHM) is a difference of the resonance angles at 50\% reflection intensity. It also shows the angular width of the reflectance curve. Detection accuracy (DA) is defined as inversely proportional to the FWHM.

$D A=\frac{1}{F W H M}\left(\right.$ Unit $\left.=1 /^{\circ}\right)($ Vibisha et al. 2020$)$

Figure of merit (FoM) indicates the multiplication of the sensitivity and DA. 
$F o M=S * D A($ Unit $=1 /$ RIU $)($ Vibisha et al. 2020)

\section{Results}

The proposed Ag-Ni-BP structure is expected to achieve considerable reflectivity and high sensitivity. The performance of the proposed SPR sensor structure in terms of reflectance and sensitivity is shown in table 1 . The corresponding various configurations, the obtained sensitivity is $143^{\circ} / \mathrm{RIU}, 120^{\circ} / \mathrm{RIU}, 129^{\circ} / \mathrm{RIU}, 135^{\circ} / \mathrm{RIU}, 284^{\circ} / \mathrm{RIU}, 347^{\circ} / \mathrm{RIU}$ as shown in fig 2 (a)-2(f), respectively. It is also shown that the highest sensitivity is achieved by using $\mathrm{Ag}(42 \mathrm{~nm})-\mathrm{Ni}$ (15nm)-BP based configuration. This enhanced sensitivity is attributed to the increased compatibility of BP with this bimetallic configuration. We have calculated the detection accuracy $\left(0.76 /^{\circ}, 0.38 /^{\circ}, 0.34 /^{\circ}, 0.31 /^{\circ}\right.$, and $\left.0.20 /^{\circ}\right)$, and FoM (91.2/RIU, 49/RIU,46.55/RIU, $88 / \mathrm{RIU}$, and 69.4/RIU) to the angular spectrum of incident light for different sensor structure as depicted in Fig.2, with and without BP layer to the variation in the sensing-medium of RI $\left(n_{s}=0.005\right)$. Hence, as noticed that with increasing the RI of the sensing-medium, the resonance angle will also shift towards the higher value. It is clear that BP is used in the SPR sensor, the resolution will be high. In fig. 4 (e), to see the effect on the reflectance curve after inserted the nickel and phosphorus layer, the resonance angle is shifted to the larger. This is due to the large real part and a small imaginary part of the dielectric constant of BP, which is attributed to the low energy loss. Based on Fig. 3(a)-(f), table 2 shows the values of resonance angle, minimum reflectance (Rmin.), sensitivity, DA, and FoM at optimized thicknesses of silver, $\mathrm{Au}, \mathrm{Ni}$, and $\mathrm{BP}$ layers.
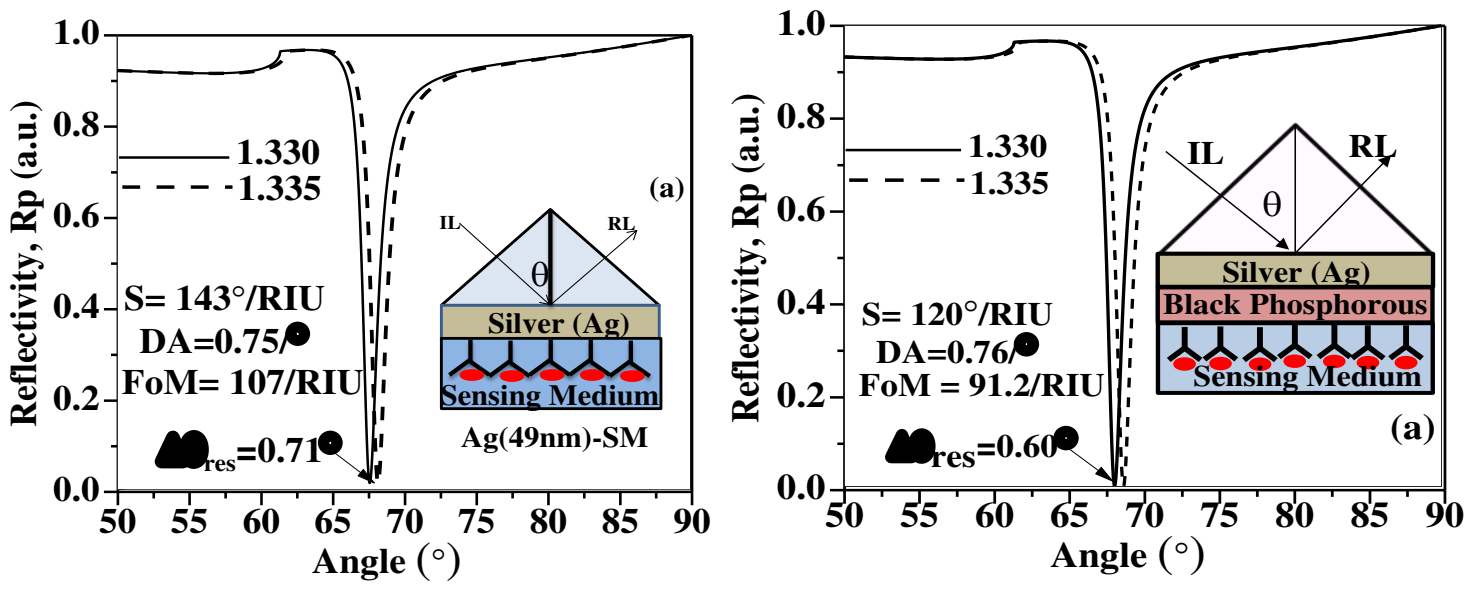

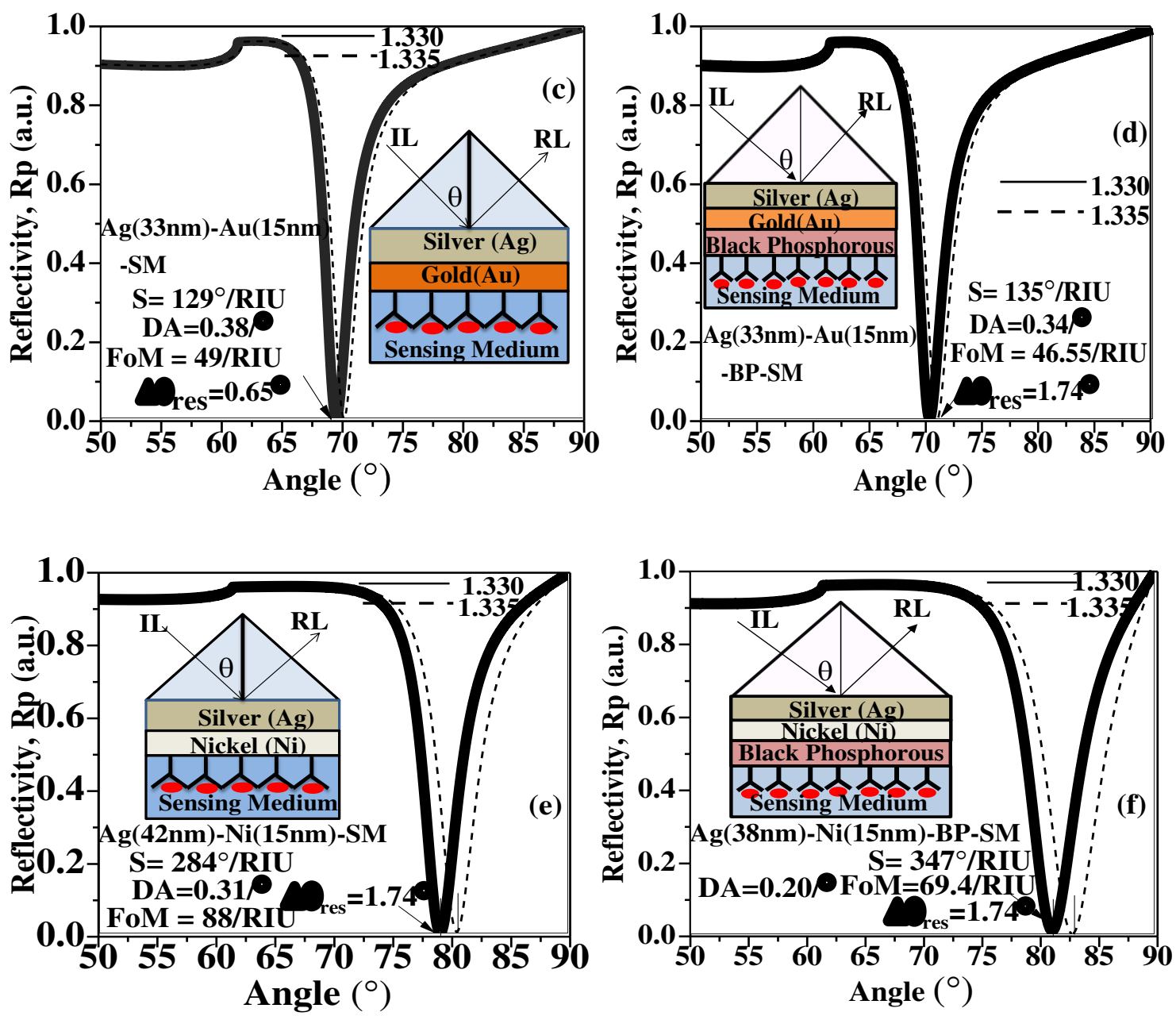

Fig. 2. Reflectance curve: (a) TraditionalSPR sensor, (b) Ag-BP based SPR sensor (c) Bimetallic (Ag-Au) Sensor (d) Bimetallic (Ag-Au) Sensor with BP (e) Bimetallic (Ag-Ni) Sensor (f) Proposed Sensor (solid line and dashed line represents as $\mathrm{n}_{\mathrm{s}}=1.330$ and 1.335 , respectively)

Table 2: Performance parameter ofAg(49nm)-SM, Ag(52nm)-BP-SM, Ag(33nm)-Au(15nm)$\mathrm{SM}, \mathrm{Ag}(33 \mathrm{~nm})-\mathrm{Au}(15 \mathrm{~nm})-\mathrm{BP}-\mathrm{SM}, \mathrm{Ag}(42 \mathrm{~nm})-\mathrm{Ni}(15 \mathrm{~nm})-\mathrm{SM}$ and proposed SPR sensor.

\begin{tabular}{|l|l|l|l|l|l|}
\hline \multicolumn{1}{|c|}{ Structure } & $\begin{array}{l}\Delta \boldsymbol{\theta}_{\text {res }} \\
\mathbf{n}_{\mathbf{s}}=\mathbf{0 . 0 0 5}\left({ }^{\circ}\right)\end{array}$ & Rmin & $\begin{array}{c}\mathbf{S} \\
(\boldsymbol{\circ} / \mathbf{R I U})\end{array}$ & $\begin{array}{l}\text { Detection } \\
\text { Accuracy }\left(/^{\circ}\right)\end{array}$ & $\begin{array}{l}\text { FoM } \\
(/ \mathbf{R I U})\end{array}$ \\
\hline Ag-SM, & 0.71 & $3.6 \times 10^{-3}$ & 144 & 0.75 & 107 \\
\hline Ag-BP-SM & 0.60 & $2.1 \times 10^{-3}$ & 120 & 0.76 & 91.2 \\
\hline Ag-Au-SM & 0.65 & $1.5 \times 10^{-6}$ & 129 & 0.38 & 49 \\
\hline Ag-Au-BP-SM & 1.74 & $2.1 \times 10^{-5}$ & 135 & 0.34 & 46.55 \\
\hline Ag-Ni-SM & 1.74 & $1 \times 10^{-4}$ & 284 & 0.31 & 88 \\
\hline Ag-Ni-BP-SM & 1.74 & $3.4 \times 10^{-4}$ & 347 & 0.20 & 69.4 \\
\hline
\end{tabular}

Using Eq. (6), we calculated the reflectivity and shift in resonance angle curves as a function of incident angle. It has a dip in resonance angle and the reflectance curve dropped sharply. Fig. 
3(a)-3(d), shows the variation of reflectivity and resonance-angle using the BP layer with an optimized thickness of silver and Nickle layers. It is found that minimum reflectivity (Rmin) firstly decreases to a certain value near to zero and then starts to increase gradually for every configuration. By comparing the Rmin for different configurations, values like $1.3 \times 10^{-4}$ a.u., $2.7 \times 10^{-4}$ a.u., $4.2 \times 10^{-4}$ a.u., $1.4 \times 10^{-6}$ a.u. for the thickness of Nickle layer $0 \mathrm{~nm}, 5 \mathrm{~nm}, 10 \mathrm{~nm} 15 \mathrm{~nm}$ and thickness of silver layer 52nm, 45nm, 39nm, 33nm as shown in Fig 3(a). Similarly, for the same Fig.3(b, c, \&d), the Rmin. is getting $2.1 \times 10^{-4}$ a.u., $6 \times 10^{-4}$ a.u., $4.2 \times 10^{-4}$ a.u., $2.1 \times 10^{-5}$ a.u., and $1.3 \times 10^{-4}$ a.u., $3.6 \times 10^{-4}$ a.u., $9 \times 10^{-4}$ a.u., $1 \times 10^{-4}$ a.u., and $2.1 \times 10^{-4}$ a.u., $1.4 \times 10^{-4}$ a.u., $3.8 \times 10^{-5}$ a.u., $3.4 \times 10^{-4}$ a.u. with the thickness of the silver layer of $52 \mathrm{~nm}, 49 \mathrm{~nm}, 46 \mathrm{~nm}, 38 \mathrm{~nm}$, and $52 \mathrm{~nm}$, $49 \mathrm{~nm}, 47 \mathrm{~nm}, 42 \mathrm{~nm}$, and $52 \mathrm{~nm}, 49 \mathrm{~nm}, 46 \mathrm{~nm}, 38 \mathrm{~nm}$, and the Nickle layer thickness of $0 \mathrm{~nm}$, $5 \mathrm{~nm}, 10 \mathrm{~nm} 15 \mathrm{~nm}$ respectively.
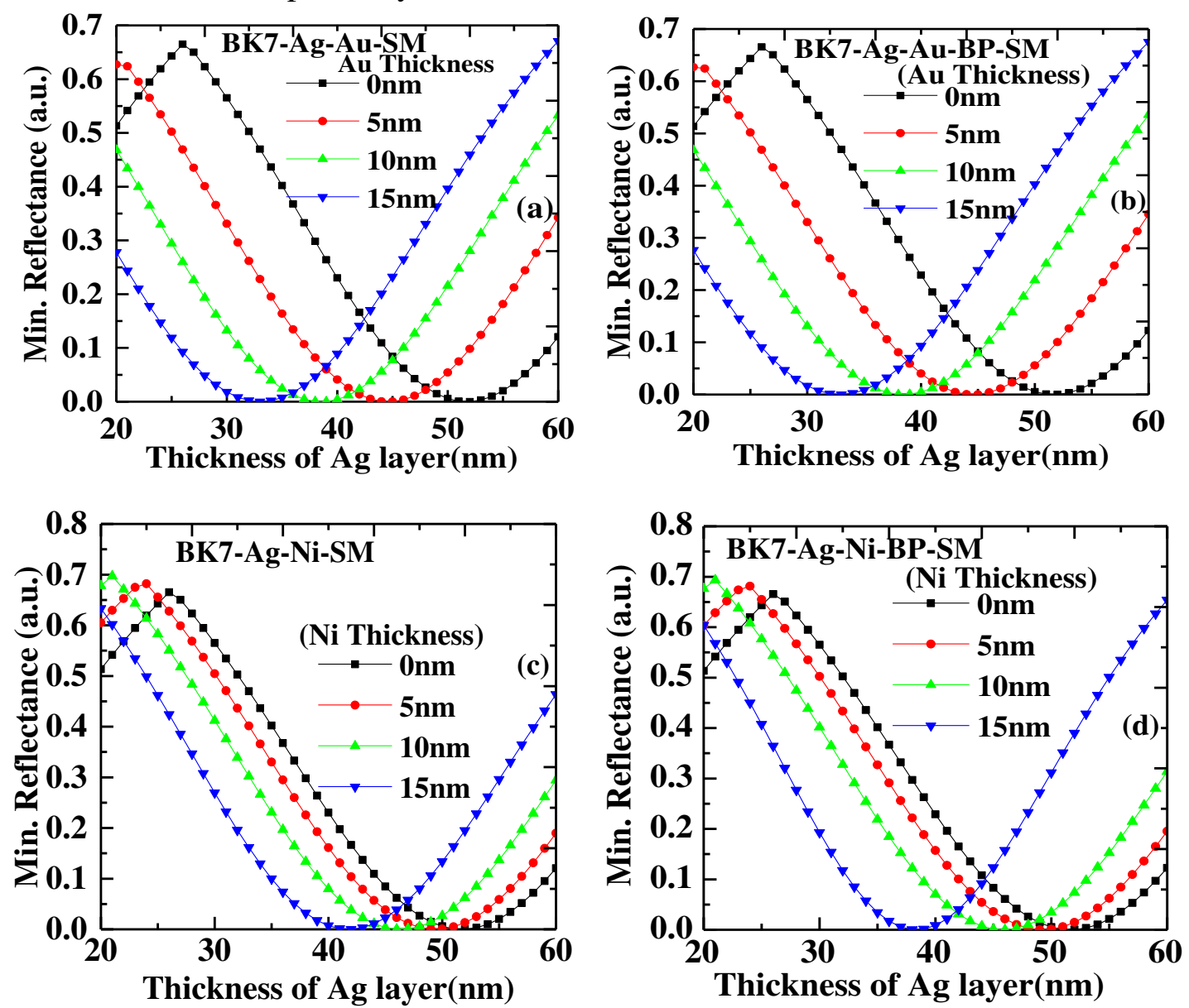

Fig. 3 Shows the Variation of $\mathrm{R}_{\min }$ at an optimized thickness of Ag layer at 20nm -60nm. (a) BK7-Ag-Au-SM (b) BK7-Ag-Au-BP-SM (c) BK7-Ag-Ni-SM (d) BK7-Ag-Ni-BP-SM, at Au and Ni layer 0 nm,5nm,10nm.15nm, respectively.

In Ag-Au-SM based SPR sensor, the obtained sensitivity at the optimized thickness of the silver layer with the thickness of an Au layer at $0 \mathrm{~nm}, 5 \mathrm{~nm}, 10 \mathrm{~nm}$, and $15 \mathrm{~nm}$ is shown in fig 4(a). It is noticed that at the calculated Rmin, the sensitivity is obtained for the given gold thickness of layer as shown in table 3. If the silver layer thickness is increased, the sensitivity is increasing up 
to increase the gold layer thickness. After adding the black phosphorus layer on the gold layer, the sensitivity is increasing up to increase the Au layer as depicted in fig. 4(b). Figure 4(a \&b), the variation of the sensitivity is ranging from $61.07^{\circ} / \mathrm{RIU}$ to $116.88^{\circ} / \mathrm{RIU}, 58.67^{\circ} / \mathrm{RIU}$ to $123.75^{\circ} / \mathrm{RIU}, 115.16^{\circ} / \mathrm{RIU}$ to $129.37^{\circ} / \mathrm{RIU}, 122.04^{\circ} / \mathrm{RIU}$ to $133.84^{\circ} / \mathrm{RIU}$, from $60.27^{\circ} / \mathrm{RIU}$ to $120.43^{\circ} / \mathrm{RIU}, 57.75^{\circ} / \mathrm{RIU}$ to $128.34^{\circ} / \mathrm{RIU}, 117.91^{\circ} / \mathrm{RIU}$ to $134.75^{\circ} / \mathrm{RIU}, 125.70^{\circ} / \mathrm{RIU}$ to $139.91^{\circ} / \mathrm{RIU}$ at the optimized thickness of silver layer with $0 \mathrm{~nm}, 5 \mathrm{~nm}, 10 \mathrm{~nm}, 15 \mathrm{~nm}$ of gold thickness layer for the structure of Ag-Au-SM and Ag-Au-BP-SM respectively. To see the effect, the sensitivity increases up to increase the thickness of gold layers at the optimized thickness of silver layer with and without BP layer.

In the proposed SPR sensor, we have replaced the gold layer with the Nickel layer at the optimized thickness of the Ag layer. The sensitivity is increased against the Ag-Au-SM and AgAu-BP-SM SPR sensor structures. The nickel layer is utilized in the SPR sensor as the same thickness as the gold layer. To improve sensitivity, the nickel layer is more consistent with the silver layer. The variation in the sensitivity is ranging from $61.07^{\circ} / \mathrm{RIU}$ to $116.88^{\circ} / \mathrm{RIU}$, $54.20^{\circ} / \mathrm{RIU}$ to $146.21^{\circ} / \mathrm{RIU}, 48.47^{\circ} / \mathrm{RIU}$ to $197.44^{\circ} / \mathrm{RIU}, 178.87^{\circ} / \mathrm{RIU}$ to $318.67^{\circ} / \mathrm{RIU}$ and without BP layer, respectively as shown in Fig. 4(c) at with $0 \mathrm{~nm}, 5 \mathrm{~nm}, 10 \mathrm{~nm}, 15 \mathrm{~nm}$ nickel layer thickness. After inserting the BP layer in the proposed structure, the sensitivity enhanced up to $347^{\circ} / \mathrm{RIU}$. Figure 4(d), the variation in sensitivity is ranging from $60.27^{\circ} / \mathrm{RIU}$ to $120.43^{\circ} / \mathrm{RIU}$, $53.17^{\circ} / \mathrm{RIU}$ to $154.69^{\circ} / \mathrm{RIU}, 47.32^{\circ} / \mathrm{RIU}$ to $221.96^{\circ} / \mathrm{RIU}, 197.21$ to $444.50^{\circ} / \mathrm{RIU}$ at the optimized thickness of silver layer with $0 \mathrm{~nm}, 5 \mathrm{~nm}, 10 \mathrm{~nm}, 15 \mathrm{~nm}$ nickel layer thickness. The sensitivity is $476^{\circ} / \mathrm{RIU}$ when the nickel layer is $20 \mathrm{~nm}$ thickness, and when the silver layer is 23nm thickness with a BP layer where the sensing- medium of RI is ranging from 1.330 to 1.335. BP has excellent property (Wu et al. 2017), due to large real and imaginary value to provide better sensitivity and more compatibility with the bimetallic combination of the Ag-Ni layer.
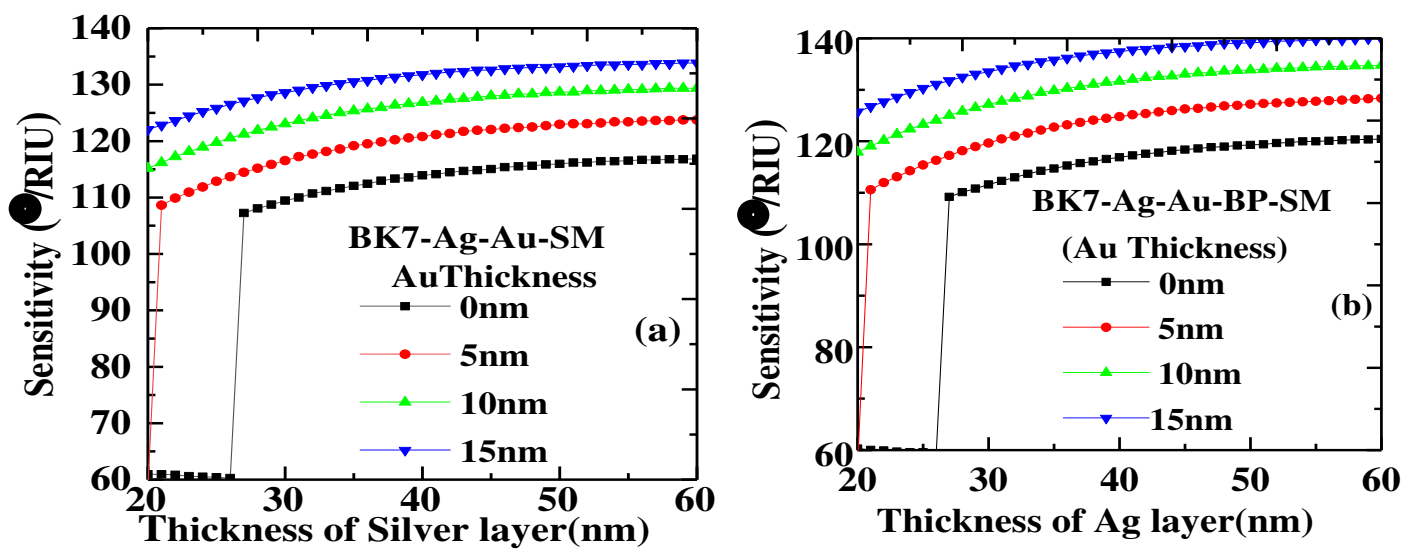

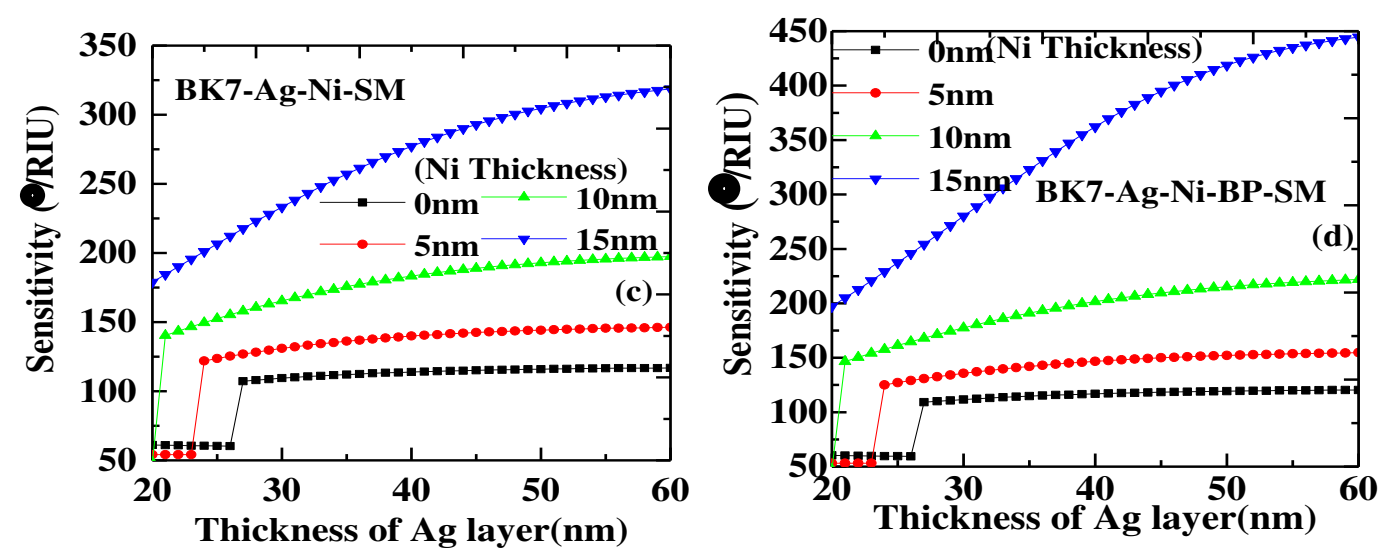

Fig.4 Shows the variation in sensitivity at optimized Cu layer thickness at 20nm -60nm. (a) BK7-Ag-Au-SM (b) BK7-Ag-Au-BP-SM (c) BK7-Ag-Ni-SM (d) BK7-Ag-Ni-BP-SM, at Au and Ni layer 0nm, 5nm, 10nm. 15nm, respectively.

In table 3, the sensitivity and Rmin calculated from the reflectance curves for the Ag (52nm)-BP-SM, Ag (33nm)-Au (15nm)-SM, Ag (33nm) - Au (15nm)-BP-SM, Ag (42nm)-Ni (15nm)-SM and the proposed SPR sensor are demonstrated for few much thicknesses variation of bimetallic layers of the $\mathrm{Ag}$ and $\mathrm{Ni}$. Table 3 is represented here to further performance analysis Ag (52nm)-BP-SM, Ag (33nm)-Au (15nm)-SM, Ag (33nm)- Au (15nm)-BP-SM, Ag (42nm)-Ni (15nm)-SM and the proposed SPR sensor, respectively at the similar combination of the Ag thicknesses (25nm, 30nm, 35nm), Au and Ni thicknesses (0nm, 15nm, 15nm).

Table 3: Sensitivity and Rmin of Ag (52nm)-BP-SM, Ag (33nm)-Au (15nm)-SM, Ag (33nm) $\mathrm{Au}$ (15nm)-BP-SM, Ag (42nm)-Ni (15nm)-SM and proposed SPR sensor for some selective Ag metal thicknesses

\begin{tabular}{|c|c|c|c|c|c|c|c|}
\hline Structure & $\begin{array}{c}\text { Ag } \\
(\mathrm{nm})\end{array}$ & $\begin{array}{c}\mathrm{Au} / \mathrm{Ni} \\
(\mathrm{nm})\end{array}$ & $1.33\left(^{\circ}\right)$ & $1.335\left(^{\circ}\right)$ & $\Delta \theta$ res & $\begin{array}{c}\text { Sensitivity } \\
\text { (\%/RIU) }\end{array}$ & $\begin{array}{l}\text { Rmin } \\
\text { (a.u.) }\end{array}$ \\
\hline \multirow{4}{*}{ BK7-Ag-Au-SM } & 52 & 0 & 67.633 & 68.214 & 0.581 & 116.2 & $1.3 \times 10^{-4}$ \\
\hline & 45 & 5 & 68.465 & 69.075 & 0.61 & 122 & $2.7 \times 10^{-4}$ \\
\hline & 39 & 10 & 69.098 & 69.731 & 0.633 & 126.6 & $4.2 \times 10^{-4}$ \\
\hline & 33 & 15 & 69.563 & 70.213 & 0.65 & 130 & $1.5 \times 10^{-6}$ \\
\hline \multirow{4}{*}{$\begin{array}{c}\text { BK7-Ag-Au-BP- } \\
\text { SM }\end{array}$} & 52 & 0 & 68.116 & 68.714 & 0.59 & 119.63 & $2.1 \times 10^{-4}$ \\
\hline & 44 & 5 & 69.021 & 69.651 & 0.62 & 125.93 & $6 \times 10^{-4}$ \\
\hline & 38 & 10 & 69.71 & 70.366 & 0.656 & 131.2 & $4 \times 10^{-4}$ \\
\hline & 33 & 15 & 70.219 & 70.894 & 0.675 & 135 & $2.1 \times 10^{-5}$ \\
\hline \multirow{3}{*}{ BK7-Ag-Ni-SM } & 50 & 5 & 70.811 & 71.532 & 0.721 & 144.2 & $6.5 \times 10^{-4}$ \\
\hline & 47 & 10 & 74.599 & 75.553 & 0.95 & 190.79 & $9 \times 10^{-4}$ \\
\hline & 42 & 15 & 79.124 & 80.544 & 1.42 & 284 & $1 \times 10^{-4}$ \\
\hline \multirow{5}{*}{$\begin{array}{c}\text { BK7-Ag-Ni-BP- } \\
\text { SM } \\
\text { (Proposed) }\end{array}$} & 52 & 0 & 68.116 & 68.714 & 0.598 & 119.6 & $2.1 \times 10^{-4}$ \\
\hline & 49 & 5 & 71.613 & 72.372 & 0.759 & 151.8 & $1.4 \times 10^{-4}$ \\
\hline & 46 & 10 & 75.926 & 76.98 & 1.054 & 210.8 & $3.8 \times 10^{-5}$ \\
\hline & 38 & 15 & 81.183 & 82.919 & 1.736 & 347 & $3.4 \times 10^{-4}$ \\
\hline & 23 & 20 & 85.127 & 87.507 & 2.38 & 476 & $1.8 \times 10^{-1}$ \\
\hline
\end{tabular}


Furthermore, to investigate the proposed sensor comparison performance parameters in terms of sensitivity, FWHM, DA, and FoMafter a tiny variation in the sensing-medium of RI the proposed sensor configuration as well as a conventional sensor as depicted in Fig. 5 (a, b, c \& d). The variation in sensitivity at Rmin is from $118.02^{\circ} / \mathrm{RIU}$ to $119.63^{\circ} / \mathrm{RIU}$, from $127.2^{\circ} / \mathrm{RIU}$ to $129.83^{\circ} / \mathrm{RIU}$, from $132.92^{\circ} / \mathrm{RIU}$ to $134.98^{\circ} / \mathrm{RIU}$, from $265.86^{\circ} / \mathrm{RIU}$ to $283.84^{\circ} / \mathrm{RIU}$, and from $315.14^{\circ} / \mathrm{RIU}$ to $347.09^{\circ} / \mathrm{RIU}$ against of $\mathrm{Ag}(52 \mathrm{~nm})-\mathrm{BP}-\mathrm{SM}, \mathrm{Ag}(33 \mathrm{~nm})-\mathrm{Au}(15 \mathrm{~nm})-\mathrm{SM}$, $\mathrm{Ag}(33 \mathrm{~nm})-\mathrm{Au}(15 \mathrm{~nm})-\mathrm{BP}-\mathrm{SM}, \mathrm{Ag}(42 \mathrm{~nm})-\mathrm{Ni}(15 \mathrm{~nm})-\mathrm{SM}$, and $\mathrm{Ag}(42 \mathrm{~nm}) \mathrm{Ni}(15 \mathrm{~nm})-\mathrm{BP}-\mathrm{SM}$ respectively, as depicted in Fig. 5(a). The increasing sensitivity is in proportion to a variation in the sensing-medium of RI. Hence, the variation in terms of FWHM is from $1.28^{\circ}$ to $1.32^{\circ}$, from $2.64^{\circ}$ to $1.74^{\circ}$, from $2.9^{\circ}$ to $3^{\circ}$, from $3.71^{\circ}$ to $4.02^{\circ}$, and from $4.98^{\circ}$ to $5.46^{\circ}$ for the same above SPR sensor configuration. The FWHM is large of the proposed SPR sensor because the RI of the real part of BP is large. Figure 5(c \& depicts the essence of DA and FoM variation as the sensing-medium of RI changes for the $\mathrm{Ag}(52 \mathrm{~nm})-\mathrm{BP}-\mathrm{SM}, \mathrm{Ag}(33 \mathrm{~nm})-\mathrm{Au}(15 \mathrm{~nm})-\mathrm{SM}, \mathrm{Ag}(33 \mathrm{~nm})$ $\mathrm{Au}(15 \mathrm{~nm})-\mathrm{BP}-\mathrm{SM}, \quad \mathrm{Ag}(42 \mathrm{~nm})-\mathrm{Ni}(15 \mathrm{~nm})-\mathrm{SM} \quad$ and $\mathrm{Ag}(42 \mathrm{~nm})-\mathrm{Ni}(15 \mathrm{~nm})-\mathrm{BP}-\mathrm{SM}$. The corresponding variation of DA is depicted in Fig. 6(c) for the $\operatorname{Ag}(52 \mathrm{~nm})-\mathrm{BP}-\mathrm{SM}, \mathrm{Ag}(33 \mathrm{~nm})$ $\mathrm{Au}(15 \mathrm{~nm})-\mathrm{SM}, \mathrm{Ag}(33 \mathrm{~nm})-\mathrm{Au}(15 \mathrm{~nm})-\mathrm{BP}-\mathrm{SM}, \mathrm{Ag}(42 \mathrm{~nm})-\mathrm{Ni}(15 \mathrm{~nm})-\mathrm{SM}$ for the proposed structure SPR sensor ranging from $0.781 /^{\circ}$ to $0.757 /^{\circ}, 0.378 /^{\circ}$ to $0.364 /^{\circ}, 0.344 /^{\circ}$ to $0.333 /^{\circ}$, $0.269 /^{\circ}$ to $0.248 /{ }^{\circ}$, and $0.200 /{ }^{\circ}$ to $0.183 /^{\circ}$, respectively. DAis analyzed for the theoretically investigated SPR biosensor is lower than that of a traditional SPR sensor. This is due to the Ni layer high imaginary dielectric constant value, which causes a damping effect due to the increased electron-loss. Because of the damping-effect, the FWHM increases, lowering DA. Similarly, the variation of FoM for the $\operatorname{Ag}(52 \mathrm{~nm})-\mathrm{BP}-\mathrm{SM}, \mathrm{Ag}(33 \mathrm{~nm})-\mathrm{Au}(15 \mathrm{~nm})-\mathrm{SM}$, $\mathrm{Ag}(33 \mathrm{~nm})-\mathrm{Au}(15 \mathrm{~nm})-\mathrm{BP}-\mathrm{SM}, \mathrm{Ag}(42 \mathrm{~nm})-\mathrm{Ni}(15 \mathrm{~nm})-\mathrm{SM}$ and the proposed structure SPR sensor is ranging from $92.20 / \mathrm{RIU}$ to $90.63 / \mathrm{RIU}, 48.18 / \mathrm{RIU}$ to $47.38 / \mathrm{RIU}, 45.83 / \mathrm{RIU}$ to $44.99 / \mathrm{RIU}$, $71.66 / \mathrm{RIU}$ to $70.60 / \mathrm{RIU}$ and 63.28 /RIU to $63.57 / \mathrm{RIU}$, respectively. It is noted that the proposed structure SPR biosensor obtains the maximum sensitivity with little decrement and increment of detection accuracy and FoM.
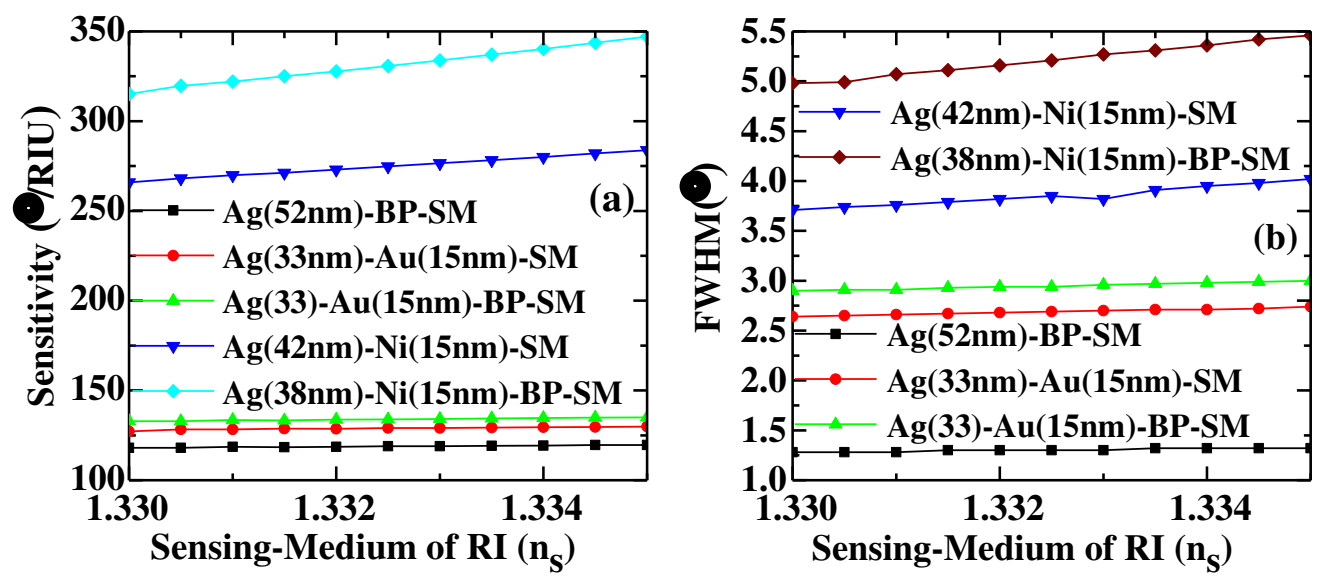

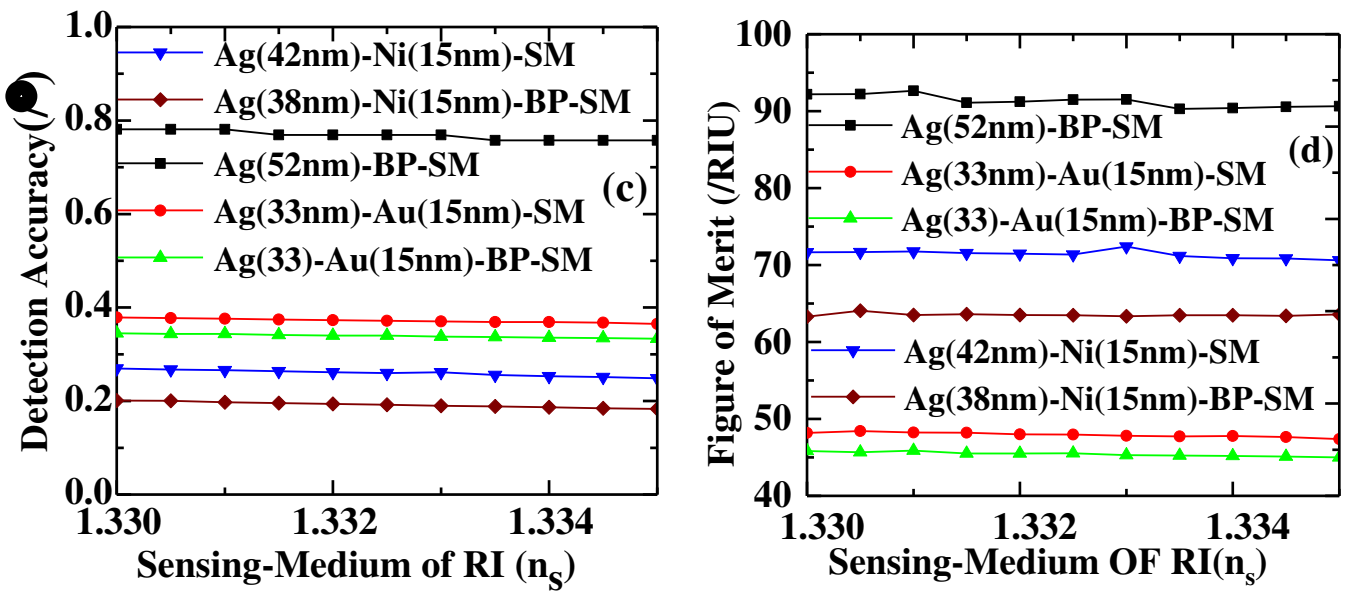

Fig. 5 Sensing-Medium of RI (ns) vs (a) Sensitivity (b) FWHM (c) DA (d) FoMat Rmin.

Finally, table 4 has shown the comparison of the proposed work and the existing SPR sensor. This work is clearly showing the maximum sensitivity other than the latest work for the traditional SPR sensor

Table 4: Performance comparison of the proposed SPR sensor with another traditional SPR sensor at $633 \mathrm{~nm}$

\begin{tabular}{|l|l|}
\hline SPR Sensor structure & $\begin{array}{l}\text { Sensitivity } \\
\left({ }^{\circ} / \text { RIU) }\right.\end{array}$ \\
\hline Prism-Au-Ti ${ }_{3} \mathrm{C}_{2} \mathrm{~T}_{\mathrm{x}}-\mathrm{Au}$-TMDs [24] & 198 \\
\hline Prism-Au- $\mathrm{Ti}_{3} \mathrm{C}_{2} \mathrm{~T}_{\mathrm{x}}-\mathrm{WS}_{2}$-Black Phosphorus [25] & 190.22 \\
\hline BK7-Ag-BP-Graphene/TMDs [26] & 279 \\
\hline Proposed work & 347 \\
\hline
\end{tabular}

\section{Conclusion:}

In this present work, we have systematically discussed the various bimetallic structure to be used in the SPR sensors. Here the application of a novel bimetallic SPR sensor with black phosphorus is discussed. The performance parameter in terms of sensitivity, FWHM, DA, FoM, and reflectivity is analyzed at the visible range. We have systematically investigated the optimized thickness of the Ag-Ni bimetallic layer and a single layer of black phosphorus. Our result indicates that when single-layer black phosphorus is grown on the above of the Ag-Ni bimetallic layer, the highest sensitivity obtained up to $347^{\circ} / \mathrm{RIU}$, which is superior to the other single and bimetallic SPR sensors. Thereafter, at 20nm thickness of Ni layer, the sensitivity is increased up to $476^{\circ} / \mathrm{RIU}$ in the proposed SPR sensor. It may be concluded that can provide remarkable enhancement of all desirable performance parameters. 


\section{References}

Biswajit Dey a, Md. Sherajul Islam a, Jeongwon Park: Numerical design of high-performance $\mathrm{WS}_{2} / \mathrm{metal} / \mathrm{WS}_{2} /$ grapheme heterostructure based surface plasmon resonance refractive index sensor Results in Physics 23 104021(2021)

Mollah MdA, Islam SMR, YousufaliMd, Abdulrazak LF, Hossain MbB, Amiri IS.: Plasmonic temperature sensor using D-shaped photonic crystal fiber Results in Physics 16:102966 (2020). 10.1016/j.rinp.2020.102966.

Moznuzzaman Md, Rafiqul Islam Md, Biplob Hossain Md, Mustafa Mehedi I.: Modeling of highly improved SPR sensor for formalin detection. Results Phys 16:102874 (2020) 10.1016/j.rinp.2019.102874.

Sharma AK, Jha R, Pattanaik HS.: Design considerations for surface Plasmon resonance based detection of human blood group in near infrared J ApplPhys 107:034701 (2010). https://doi.org/10.1063/1.3298503.

Lam WW, Chu LH, Wong CL, Zhang YT.: A surface plasmon resonance system for the measurement of glucose in aqueous solution Sens Actuators, B 105: 138-43 (2005) https://doi.org/10.1016/j.snb.2004.04.088.

Shankaran D, R, Gobi K.V, Miura N.: Recent advancements in surface plasmon resonance immune-sensors for detection of small molecules of biomedical, food and environmental interest Sens Actuators, B 121:158-177 (2007) https://doi.org/ 10.1016/j.snb.2006.09.014.

Mishra SK, Tripathi SN, Choudhary V, Gupta BD.: SPR based fiber optic ammonia gas sensor utilizing nanocomposite film of PMMA/reduced graphene oxide prepared by in situ $\begin{array}{lllll}\text { polymerization } & \text { Sens } \quad \text { Actuators, } & \text { B } & 199: 190-200 & \text { (2014). }\end{array}$ https://doi.org/10.1016/j.snb.2014.03.109.

Maurya JB, Prajapati YK, Tripathi R.: Effect of molybdenum disulfide layer on surface plasmon resonance biosensor for the detection of bacteria Silicon 10: 245-56 (2018). https://doi.org/10.1007/s12633-016-9431-y.

Menon PS, Said FA, Mei GS, Berhanuddin DD, Umar AA, Shaari S, et al.: Urea and creatinine detection on nano-laminated gold thin film using Kretschmann-based surface plasmon

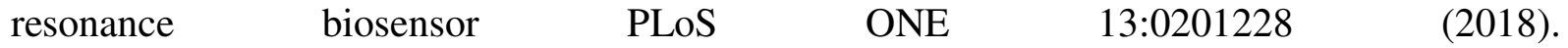
https://doi.org/10.1371/journal.pone.0201228.

Rahman MS, Anower MS, Rahman MK, Hasan MR, Hossain MB, Haque MI.: Modeling of a highly sensitive MoS2-Graphene hybrid based fiber optic SPR biosensor for sensing DNA hybridization Optik 140:989-97 (2017) https://doi.org/10.1016/j.ijleo.2017.05.001.

Yanase Y, Yoshizaki K, Kimura K, Kawaguchi T, Hide M, Uno S.: Development of SPR imaging-impedance sensor for multi-parametric living cell analysis Sensors 19:2067 (2019). https://doi.org/10.3390/s19092067

Yuan, Y., Yu, X., Ouyang, Q., Shao, Y., Song, J., Qu, J., \& Yong, K. T. Highly anisotropic black phosphorous-graphene hybrid architecture for ultrasensitive plasmonic biosensing Theoretical insight 2D Materials, 5(2), 025015 (2018).

Wu, L., Jia, Y., Jiang, L., Guo, J., Dai, X., Xiang, Y. and Fan, D.: Sensitivity improved SPR biosensor based on the $\mathrm{MoS}_{2}$ /graphene-aluminum hybrid structure Journal of Lightwave Technology, 35(1), 82-87 (2016). 
Kumar R, Kushwaha AS, Srivastava M, Mishra H, Srivastava SK.: Enhancement in sensitivity of graphen base zinc oxide assisted bimetallic surface Plasmon resonance (SPR) biosensor Appl. Physs. A 124(3);235 (2018).

Shalabney A, Abdulhalim I.: Sensitivity-enhancement methods for surface Plasmon sensors: SPR sensors sensitivity enhancement Laser \& Photon Rev 5: 571-606 (2011). https://doi.org/10.1002/lpor.201000009.

Dyankov G, Zekriti M, Bousmina M.: Dual-mode surface-plasmon sensor based on bimetallic film Appl Opt 51:2451 (2012). https://doi.org/10.1364/AO.51.002451.

Kashyap R, Chakraborty S, Zeng S, Swarnakar S, Kaur S, Doley R.: Enhanced biosensing activity of bimetallic surface plasmon resonance sensor Photonics 6:108 (2019). https://doi.org/10.3390/photonics6040108.

Chen S, Lin C.: High-performance bimetallic film surface plasmon resonance sensor based on film thickness $\quad$ optimization $\quad$ Optik $\quad 127: 7514-9 \quad$ (2016) https://doi.org/10.1016/j.ijleo.2016.05.085.

M. Mitsushio, K. Miyashita, M. Higo.: Sensor properties and surface characterization of the metal-deposited SPR optical fiber sensors with $\mathrm{Au}, \mathrm{Ag}, \mathrm{Cu}$, and $\mathrm{Al}$, Sens Actuators A 125 296-303 (2006).

A.A. Rifat, G.A. Mahdiraji, R. Ahmed, D.M. Chow, Y.M. Sua, Y.G. Shee, F.R.M. Adikan.: Copper-graphene-based photonic crystal fiber plasmonic biosensor IEEE Photon. J 8 4800408 (2016).

Wang, Q. H.; Kalantar-Zadeh, K.; Kis, A.; Coleman, J. N.; Strano, M. S.: Electronics and optoelectronics of two-dimensional transition metal dichalcogenides. Nat. Nanotechnol. 7, 699-712 (2012).

Abbas, A. N.; Liu, B.; Chen, L.; Ma, Y.; Cong, S.; Aroonyadet, N.; Köpf, M.; Nilges, T.; Zhou, C.: Black phosphorus gas sensors Acs Nano 9, 5618-5624 (2015).

Yasaei, P.; Kumar, B.; Foroozan, T.; Wang, C.; Asadi, M.; Tuschel, D.; Indacochea, J. E.; Klie, R. F.; Salehi-Khojin.: A High-quality black phosphorus atomic layers by liquid-phase exfoliation Adv. Mater. 27, 1887-1892 (2015)

Sharma, A.K. and Gupta, B.D.: On the performance of different bimetallic combinations in surface plasmon resonance based fiber optic sensors Journal of applied physics, 101(9), 093111(2007)

Srivastava, A., Verma, A., Das, R., \& Prajapati, Y. K. : A theoretical approach to improve the performance of SPR biosensor using MXene and black phosphorus Optik, 203, 163430 (2020)

Alagu Vibisha, G., Nayak, J.K., Maheswari, P., Priyadharsini, N., Nisha, A., Jaroszewicz, Z., Rajesh, K.B. and Jha, R.: Sensitivity enhancement of surface plasmon resonance sensor using hybrid configuration of 2D materials over bimetallic layer of $\mathrm{Cu}-\mathrm{Ni}$ Optics Communications, 463, 125337 (2020).

Wu, L., Guo, J., Wang, Q., Lu, S., Dai, X., Xiang, Y. and Fan, D.: Sensitivity enhancement by using few-layer black phosphorus-graphene/TMDCs heterostructure in surface plasmon resonance biochemical sensor Sensors and Actuators B: Chemical, 249, 542-548(2017)

Maharana, P.K. and Jha, R.: Chalcogenide prism and graphene multilayer based surface plasmon resonance affinity biosensor for high performance. Sensors and Actuators B: Chemical, 169, 161-166 (2012). 
Figures

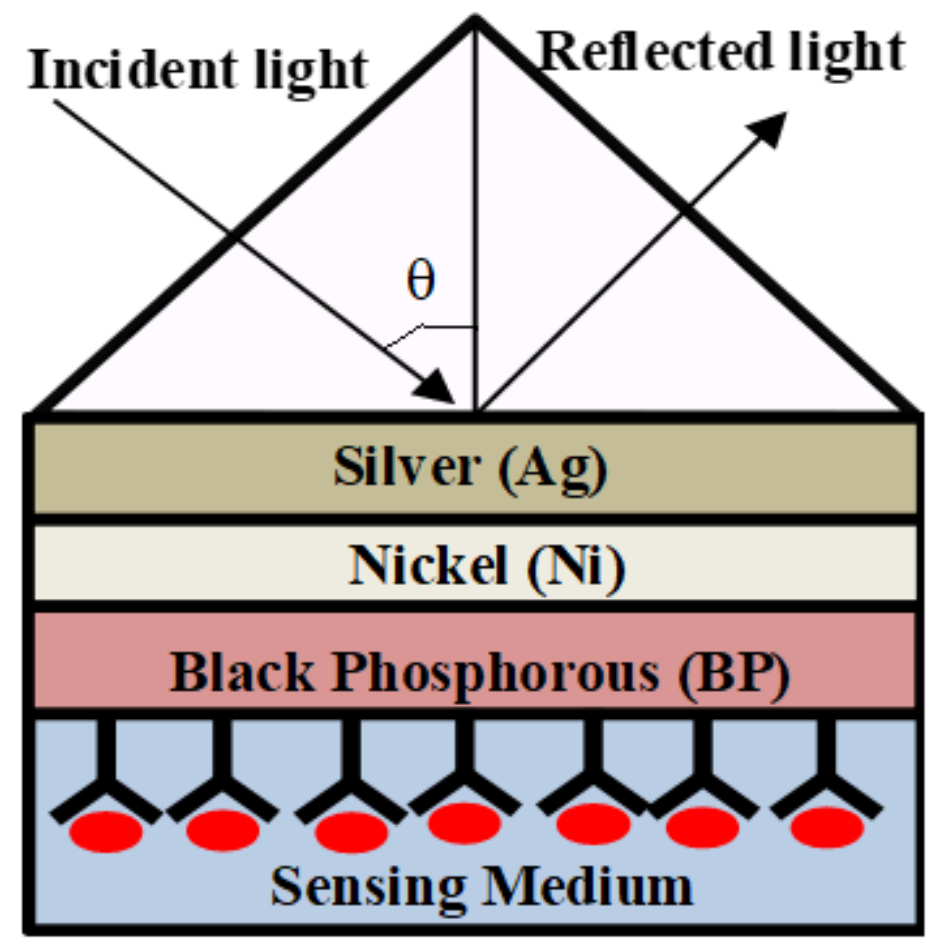

Figure 1

Proposed SPR Sensor 

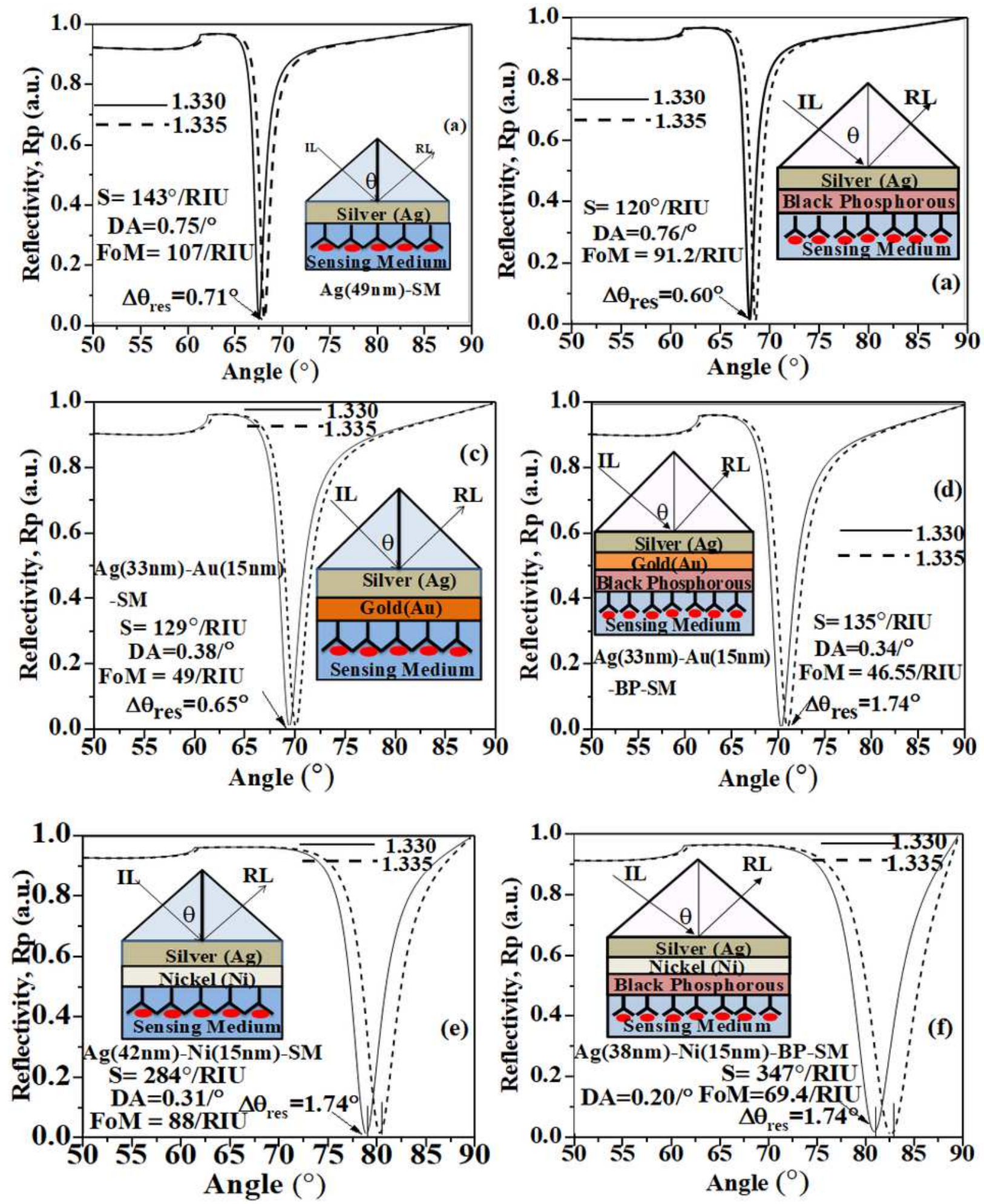

Figure 2

Reflectance curve: (a) TraditionalSPR sensor, (b) Ag-BP based SPR sensor (c) Bimetallic (Ag-Au) Sensor (d) Bimetallic (Ag-Au) Sensor with BP (e) Bimetallic (Ag-Ni) Sensor (f) Proposed Sensor (solid line and dashed line represents as $n s=1.330$ and 1.335 , respectively) 

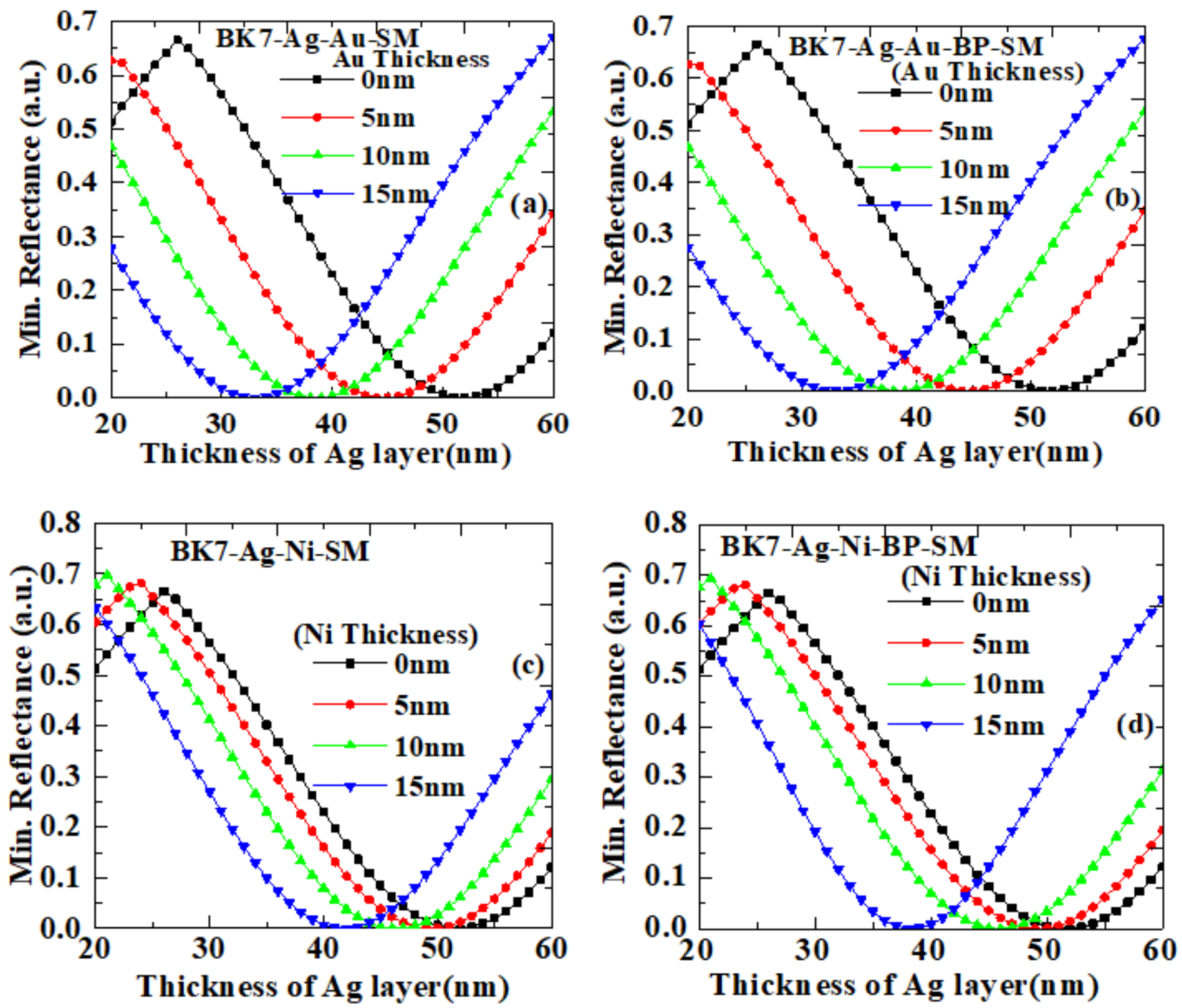

Figure 3

Shows the Variation of Rmin at an optimized thickness of Ag layer at 20nm -60nm. (a) BK7-Ag-Au-SM (b) BK7-Ag-Au-BP-SM (c) BK7-Ag-Ni-SM (d) BK7-Ag-Ni-BP-SM, at Au and Ni layer $0 \mathrm{~nm}, 5 \mathrm{~nm}, 10 \mathrm{~nm} .15 \mathrm{~nm}$, respectively. 

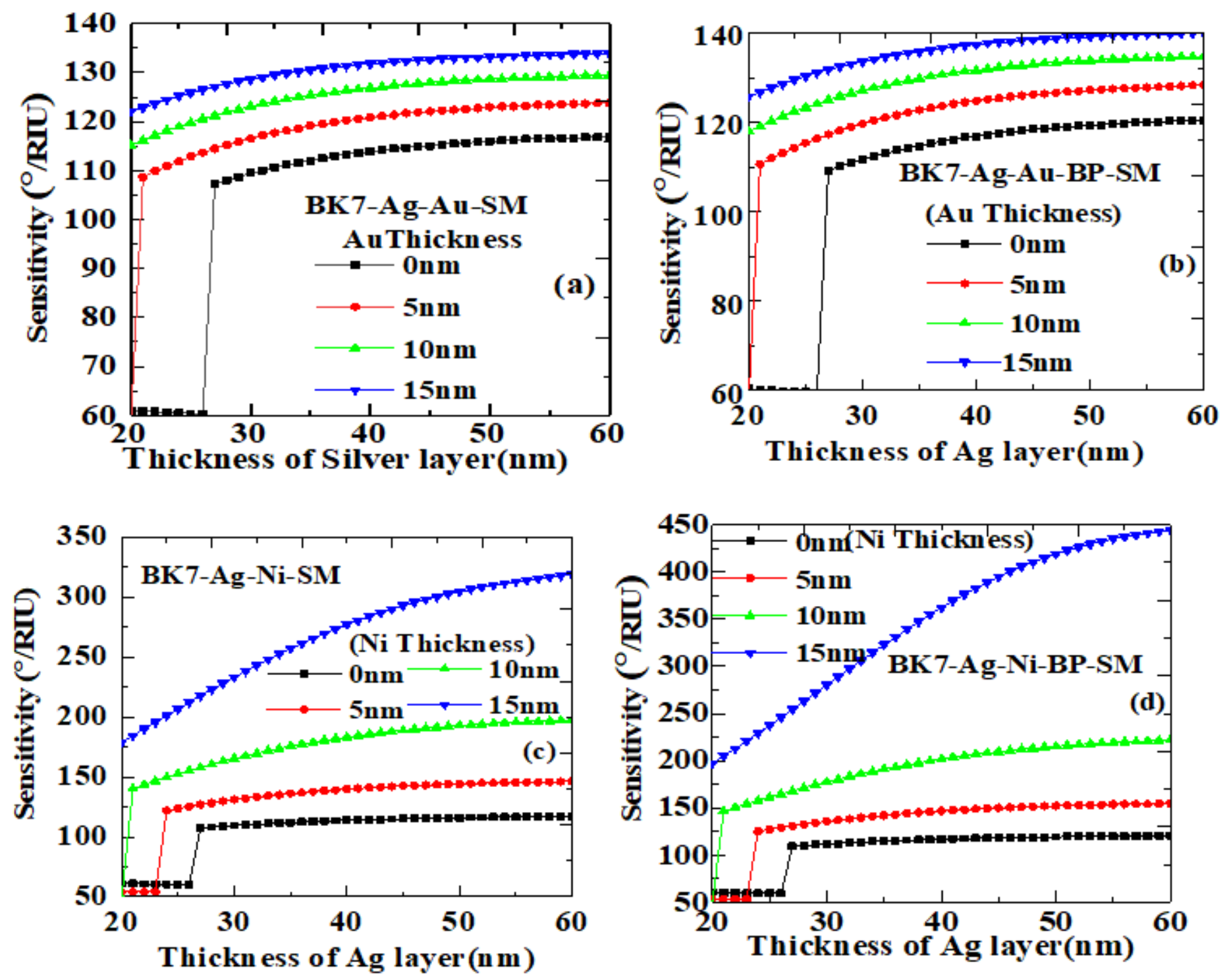

Figure 4

Shows the variation in sensitivity at optimized Cu layer thickness at 20nm -60nm. (a) BK7-Ag-Au-SM (b) BK7-Ag-Au-BP-SM (c) BK7-Ag-Ni-SM (d) BK7-Ag-Ni-BP-SM, at Au and Ni layer Onm, 5nm, 10nm. 15nm, respectively. 

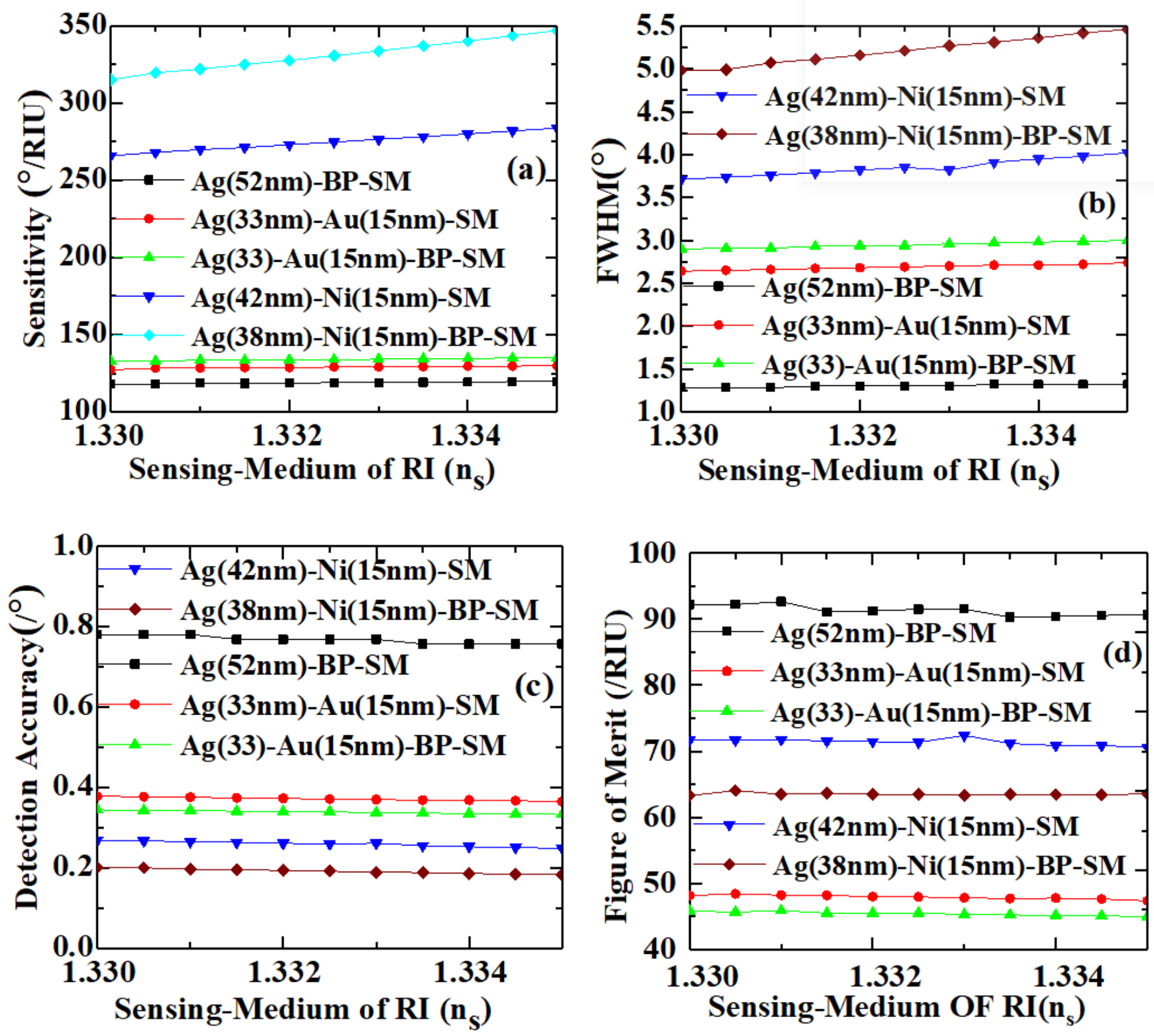

Figure 5

Sensing-Medium of RI (ns) vs (a) Sensitivity (b) FWHM (c) DA (d) FoMat Rmin. 